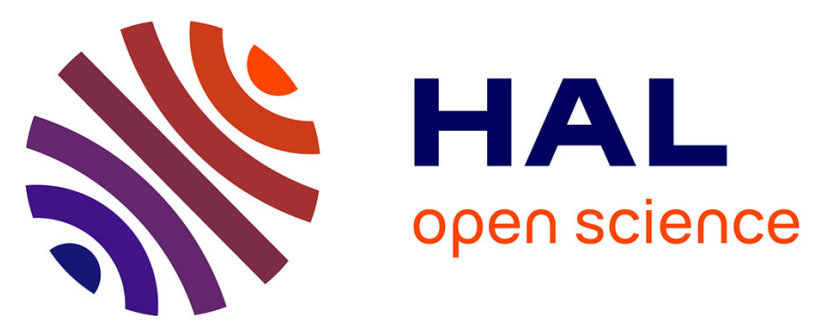

\title{
Global Linear Stability Analysis of the Flow Around a Superhydrophobic Circular Cylinder
}

Franco Auteri, Marco Carini, Michel Fournié, Dominique Fratantonio, Flavio

Giannetti

\section{- To cite this version:}

Franco Auteri, Marco Carini, Michel Fournié, Dominique Fratantonio, Flavio Giannetti. Global Linear Stability Analysis of the Flow Around a Superhydrophobic Circular Cylinder. Springer Proceedings in Physics, 2016, Proceedings of the 5th International Conference on Jets, Wakes and Separated Flows (ICJWSF2015), 185, pp.165-170. 10.1007/978-3-319-30602-5_21. hal-01978092

\section{HAL Id: hal-01978092 \\ https://hal.science/hal-01978092}

Submitted on 22 Jan 2019

HAL is a multi-disciplinary open access archive for the deposit and dissemination of scientific research documents, whether they are published or not. The documents may come from teaching and research institutions in France or abroad, or from public or private research centers.
L'archive ouverte pluridisciplinaire HAL, est destinée au dépôt et à la diffusion de documents scientifiques de niveau recherche, publiés ou non, émanant des établissements d'enseignement et de recherche français ou étrangers, des laboratoires publics ou privés. 


\title{
Global Linear Stability Analysis of the Flow Around a Superhydrophobic Circular Cylinder
}

\author{
Franco Auteri, Marco Carini, Michel Fournié, Dominique Fratantonio \\ and Flavio Giannetti
}

\begin{abstract}
Over the last few years, superhydrophobic $(\mathrm{SH})$ surfaces have been receiving an increasing attention in many scientific areas by virtue of their ability to enhance flow slip past solid walls and reduce the skin-friction drag. In the present study, a global linear-stability analysis is employed to investigate the influence of the SH-induced slip velocity on the primary instability of the 2D flow past a circular cylinder. The flow regions playing the role of 'wavemaker' are identified by considering the structural sensitivity of the unstable mode, thus highlighting the effect of slip on the global instability of the considered flow. In addition, a sensitivity analysis to slip-induced base-flow modifications is performed, revealing which areas of the cylinder surface provide a stabilising/destabilising effect when treated with a SH coating.
\end{abstract}

\section{Introduction}

The non-adhesive property of superhydrophobic ( $\mathrm{SH}$ ) surfaces originates from the presence of a non-wetted contact that holds when a low-viscosity gas is trapped inside the blemishes of the solid surface. From a macroscopical viewpoint, this phenomenon can be mathematically described by substituting the standard no-slip

\author{
F. Auteri $(\bowtie) \cdot D$. Fratantonio \\ Dipartimento di Scienze e Tecnologie Aerospaziali, Politecnico di Milano, \\ via La Masa 34, 20156 Milano, Italy \\ e-mail: franco.auteri@polimi.it \\ M. Carini \\ Institut de Mécanique des Fluides de Toulouse, Allée du Professeur Camille Soula, \\ 31400 Toulouse, France \\ M. Fournié \\ Institut de Mathématiques de Toulouse, Université Paul Sabatier, 118 route de Narbonne, \\ 31062 Toulouse, France \\ F. Giannetti \\ Dipartimento di Ingegneria Industriale, Università degli Studi di Salerno, \\ Via Giovanni Paolo II 132, 84084 Fisciano, Italy \\ (C) Springer International Publishing Switzerland 2016 \\ A. Segalini (ed.), Proceedings of the 5th International Conference \\ on Jets, Wakes and Separated Flows (ICJWSF2015), \\ Springer Proceedings in Physics 185, DOI 10.1007/978-3-319-30602-5_21
}


boundary condition in favor of the so called Navier condition [1], which can be expressed in non-dimensional form as:

$$
\mathbf{U} \cdot \hat{\boldsymbol{\tau}}=-\ell\left[\left(\nabla \mathbf{U}+\nabla \mathbf{U}^{T}\right) \cdot \hat{\mathbf{n}}\right] \cdot \hat{\boldsymbol{\tau}}, \quad \text { on } \quad \Gamma_{c y l},
$$

where $\hat{\boldsymbol{\tau}}$ and $\hat{\mathbf{n}}$ are the unit vectors tangent and normal to the cylinder surface $\Gamma_{c y l}$, respectively, $\mathbf{U}$ denotes the flow velocity made dimensionless with the incoming uniform flow velocity, and $\ell$ is the slip length made dimensionless with the diameter $D$ of the cylinder.

Given that, to the best of the authors' knowledge, the highest slip length experimentally achieved with a stable air-liquid interface is $\sim 400 \mu \mathrm{m}$ [2], the diameter of the cylinder must be on the order of tens of millimeters if we want $\mathrm{SH}$ coatings to have noticeable effects on the flow dynamics. Therefore, the present analysis provides results that are useful for the exploitation of $\mathrm{SH}$ surfaces as control devices in microfluidic systems, e.g., for flow mixing control.

Among all the earlier studies on the subject [3-7], Legendre et al. [3] have investigated the influence of a uniform slip length on the two-dimensional wake of a circular cylinder by means of Direct Numerical Simulation (DNS). Legendre et al. [3] traced a stability diagram in the parameter space $(\ell, \mathrm{Re})$, indicating that a complete suppression of the vortex shedding can be obtained for $\ell \geq 0.25$. Similarly, Mastrokalos et al. [4] carried out DNSs for piecewise-constant slip-length distributions, showing that applying SH coating in the rear and the front stagnation point regions has a destabilising effect or no effect at all on the Bénard-Von Kármán instability.

Starting from past studies, which mainly consist in numerical simulations, the present work aims to shed light on the phenomenology behind the stabilising effect given by SH coatings. In order to do so, we analyse how the base flow, the critical direct and adjoint modes, and the sensitivity of the leading eigenvalue change at increased levels of slip while moving along the neutral curve in the parameter space $(\ell, \mathrm{Re})$. This investigation is supported by a sensitivity analysis to slip-length modifications and slip-induced base-flow modifications, which gives useful indications on the role played by the slip.

\section{Theoretical Framework}

The adopted computational domain $\Omega$ is a two-dimensional box, whose boundaries are the cylinder surface $\Gamma_{c y l}$, the inflow boundary $\Gamma_{i n}$, the top and bottom boundaries $\Gamma_{\text {lat }}$ and the outflow boundary $\Gamma_{\text {out }}$. Within $\Omega$, the fluid motion is described by the usual two-dimensional unsteady, incompressible Navier-Stokes (NS) equations supplemented by the following boundary conditions:

$$
\begin{aligned}
& \mathbf{U}=\hat{\mathbf{x}}, \quad \text { on } \quad \Gamma_{i n}, \\
& \mathbf{U} \cdot \hat{\mathbf{n}}=0, \quad \text { on } \quad \Gamma_{c y l}, \\
& \mathbf{U} \cdot \hat{\boldsymbol{\tau}}=-\ell\left[\left(\nabla \mathbf{U}+\nabla \mathbf{U}^{T}\right) \cdot \hat{\mathbf{n}}\right] \cdot \hat{\boldsymbol{\tau}}, \text { on } \Gamma_{c y l},
\end{aligned}
$$




$$
P \hat{\mathbf{n}}-\frac{1}{\operatorname{Re}}\left(\nabla \mathbf{U}+\nabla \mathbf{U}^{T}\right) \cdot \hat{\mathbf{n}}=\mathbf{0}, \quad \text { on } \quad \Gamma_{\text {out }} \text { and } \Gamma_{\text {lat }},
$$

where $\mathbf{U}$ and $P$ are the velocity and the pressure of the flow, respectively. The Reynolds number is defined as $\operatorname{Re}=U_{\infty} D / \nu$, with $U_{\infty}$ being the free-stream velocity, $v$ the kinematic viscosity of the fluid, $\hat{\mathbf{x}}$ is the unit vector of the $x$ axis, and $D$ the cylinder diameter.

The linear stability analysis is carried out by decomposing the flow field in a steady part and a small unsteady perturbation $(\hat{\mathbf{u}}(x, y, t), \hat{p}(x, y, t))$, with the ansatz $\hat{\mathbf{u}}(x, y, t)=\mathbf{u}(x, y) \exp (\gamma t)$ and $\hat{p}(x, y, t)=p(x, y) \exp (\gamma t)$ where $\gamma$ is a complex number (see for instance [8]).

\section{Numerical Approach and Code Validation}

The numerical approach is based on a finite element method applied on a mesh made of triangular elements, which has been implemented by means of the GetFem++ library [9]. In the present work, the weak formulation of the linearized NS equations involves the use of the Lagrange-multiplier technique to impose the nopenetration boundary condition, while the slip boundary condition is introduced in the overall weak formulation as commonly done for a Robin condition. The MUMPS library [10], the ARPACK library [11], and the LOCA library [12] are employed to solve the involved algebraic linear systems, the eigenvalue problems, and to track the Hopf bifurcation, respectively. The code has been extensively validated for either no-slip, uniform slip, or piecewise-constant slip boundary conditions. Considering slip-length values up to 0.2 and Reynolds numbers less than 200, we have continued the Hopf bifurcation in the parameter space $(\ell, \mathrm{Re})$. Figure 1 shows how the

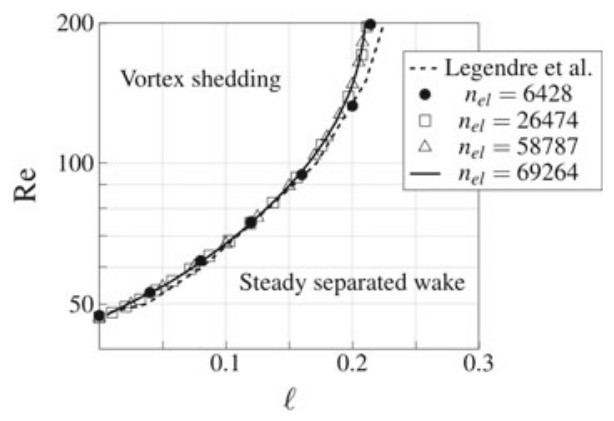

Fig. 1 Hopf-bifurcation neutral curve in the parameter space $(\ell, \mathrm{Re})$. The dashed line represents the extrapolated results of Legendre et al. [3]. The solid black line and the marks represent the bifurcation boundary traced with the continuation algorithm in the present work. In the legend, $n_{e l}$ denotes the number of triangular elements employed. A logarithmic scale is adopted for the Reynolds axis 
neutral curve is almost insensitive to the employed mesh refinement. The obtained results match very well the stability diagram traced by Legendre et al. [3]. The case of piecewise-constant slip-length distribution has also been tested by considering some of the distributions employed by Mastrokalos et al. [4] and by verifying that the results of our global linear stability analysis are consistent with their DNSs.

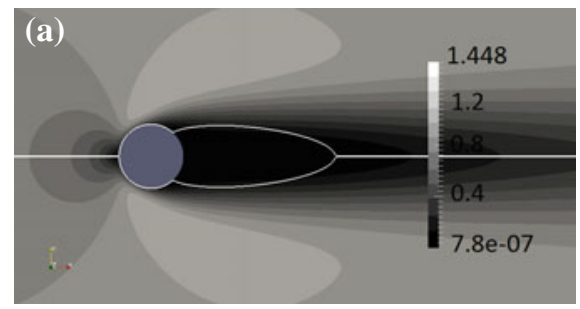

$\ell_{c}=0.05, \operatorname{Re}_{c}=54.77$

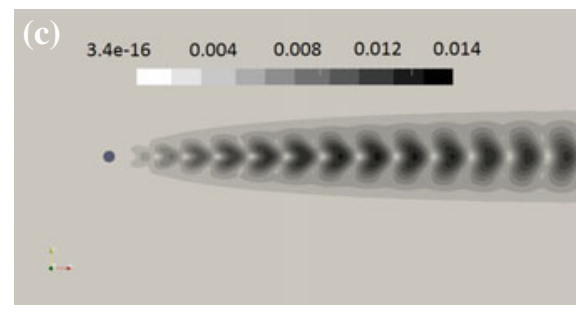

$\ell_{c}=0.05, \operatorname{Re}_{c}=54.77$

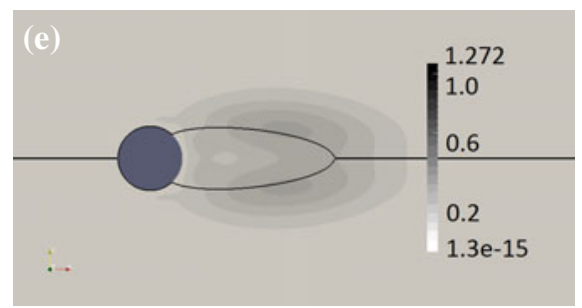

$\ell_{c}=0.05, \operatorname{Re}_{c}=54.77$

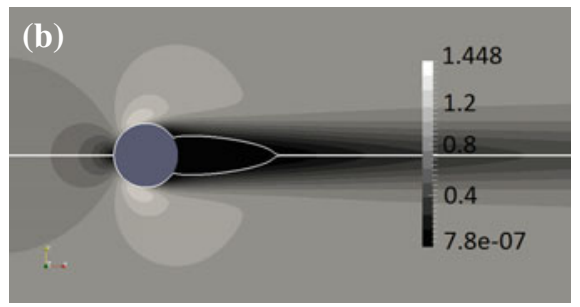

$\ell_{c}=0.2, \operatorname{Re}_{c}=147.85$

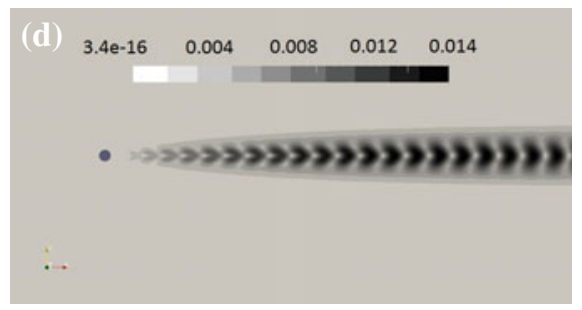

$\ell_{c}=0.2, \operatorname{Re}_{c}=147.85$

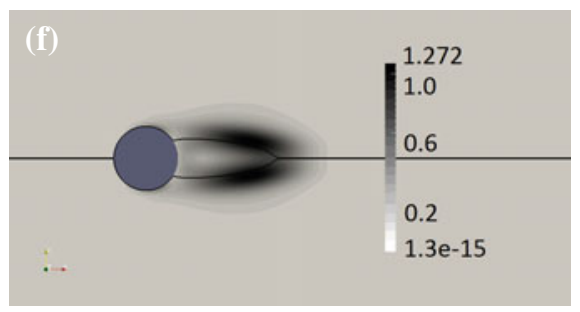

$\ell_{c}=0.2, \operatorname{Re}_{c}=147.85$

Fig. 2 Variation of the flow properties along the neutral curve: $\mathbf{a}, \mathbf{b}$ velocity magnitude of the base flow; $\mathbf{c}, \mathbf{d}$ velocity magnitude of the direct mode; $\mathbf{e}, \mathbf{f}$ structural sensitivity function

\section{Results and Discussion}

The influence of the slip on the instability phenomenon is investigated by analysing how the flow properties change along the bifurcation boundary. Figure 2 illustrates the base flow, the direct eigenmode and the structural sensitivity function as defined in Giannetti and Luchini [8] for two points on the neutral curve of Fig. 1, namely at 

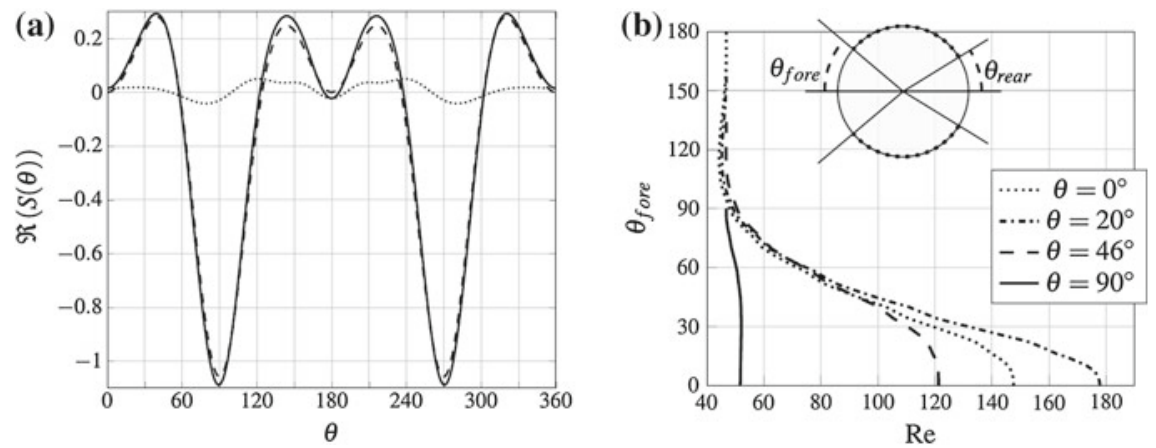

Fig. 3 a Sensitivity function to (dotted line) slip-length modification $S_{p}$ and (dashed line) slipinduced base-flow modifications $S_{b}$ as a function of the angular position on the cylinder surface. The solid line represents the overall effect given by the sum of the two functions. $\mathbf{b}$ Neutral curves in the parameter space $\left(\theta_{\text {fore }}, \mathrm{Re}\right)$ for $\theta_{\text {rear }}=0^{\circ}, 20^{\circ}, 46^{\circ}, 90^{\circ}$ and $\ell=0.2$. The rough areas on the cylinder sketch represent the presence of slip, while the smooth areas are no-slip surfaces

$\ell=0.05$ and $\ell=0.2$. As the recirculation bubble of the steady wake gets smaller at increased levels of slip, the direct mode and the structural sensitivity function follow the same trend. Indeed, the instability cores become smaller and more intense, but their position relative to the boundary of the recirculation bubble remains unchanged at increased levels of slip. These observations suggest that the nature of the instability is not modified by the slip. Moreover, we can infer from Fig. 2c, $d$ that the wavenumber of the spatial oscillation of the direct mode increases with the slip, coherently with the increase of the critical Strouhal number.

A slip-length modification results in a double effect on the eigenvalue problem. A localized perturbation of the slip-length $\delta \ell(\theta)$ modifies both the boundary conditions of the eigenvalue problem and the boundary conditions of the base-flow equations [13]. By exploiting the Lagrange identity and the solution of the adjoint eigenvalue problem, we derive two sensitivity functions, i.e., $S_{p}$, the sensitivity related to the direct modification of the eigenvalue problem, and $S_{b}$, the sensitivity related to the induced base-flow modifications, whose expressions are:

$$
S_{p}(\theta)=\frac{\hat{u}_{\hat{\boldsymbol{\tau}}}^{\dagger *}(\theta) \hat{u}_{\hat{\boldsymbol{\tau}}}(\theta)}{\left(\hat{\mathbf{u}}^{\dagger}, \hat{\mathbf{u}}\right)_{\Omega} \ell^{2}(\theta) \operatorname{Re}}, \quad S_{b}(\theta)=\frac{U_{b, \hat{\boldsymbol{\tau}}}^{\dagger *}(\theta) U_{b, \hat{\tau}}(\theta)}{\left(\hat{\mathbf{u}}^{\dagger}, \hat{\mathbf{u}}\right)_{\Omega} \ell^{2}(\theta) \operatorname{Re}} .
$$

In (3), both sensitivity functions are given by the product between the tangential component of two velocity fields, where $\mathbf{U}_{b}$ is the base flow, $\hat{\mathbf{u}}$ and $\hat{\mathbf{u}}^{\dagger}$ are the critical direct and adjoint modes, respectively, and $\mathbf{U}_{b}^{\dagger}$ is the adjoint base flow, as defined in Marquet et al. [14]. As illustrated in Fig. 3a, the application of the above sensitivity analysis to the case $\ell=0$ shows that slip surfaces applied on the flanks of the cylinder surface, namely in the range $\theta=[60,120]$, provide a stabilising effect, while the front and rear areas provide a destabilization of the base flow. These observations are 
in good agreement with the numerical simulations carried out by Mastrokalos et al. [4]. The same conclusions can be inferred from the neutral curves in the parameter space $\left(\theta_{\text {fore }}, \mathrm{Re}\right)$ computed at different values of $\theta_{\text {rear }}$, where $\theta_{\text {fore }}$ and $\theta_{\text {rear }}$ define the no-slip areas in the front and the rear of the cylinder surface, respectively. According to Fig. 3b, the critical Reynolds number can be further increased if the slip surfaces are removed from the rear-stagnation-point area.

\section{Summary and Conclusions}

Our global linear stability analysis explains how the presence of slip delays the onset of instability and increases the critical Reynolds and Strouhal numbers. The structural-sensitivity analysis at the perturbation level [8] suggests that the flow slip does not modify the instability mechanisms. Moreover, the sensitivity analysis with respect to slip-length modifications and slip-induced base-flow modifications reveals that only slip surfaces applied on the flanks of the cylinder can delay the Von Kármán instability. This observation is further supported by the neutral curves in the parameter space $\left(\theta_{\text {fore }}, \mathrm{Re}\right)$, which show an increase of the critical Reynolds number for increasing values of $\theta_{\text {rear }}$ in the neighbourhood of $\theta_{\text {rear }}=0^{\circ}$.

\section{References}

1. C.L.M.H. Navier, Mémoires de l'Académie Royale des Sciences de l'Institut de France 6, 389$440(1823)$

2. C. Lee, C.J. Kim, Langmuir 25(21), 12812-12818 (2009)

3. D. Legendre, E. Lauga, J. Magnaudet, J. Fluid Mech. 633, 437-447 (2009)

4. E.M. Mastrokalos, C.I. Papadopoulos, L. Kaiktsis, Comput. Fluids 107, 256-271 (2015)

5. P. Muralidhar, N. Ferrer, R. Daniello, J.P. Rothstein, J. Fluid Mech. 680, 459-476 (2011)

6. D. Li, S. Li, Y. Xue, Y. Yang, W. Su, Z. Xia, Y. Shi, H. Lin, H. Duan, J. Fluids Struct. 51, 211-224 (2014)

7. D. You, P. Moin, Phys. Fluids 19, 081701 (2007)

8. F. Giannetti, P. Luchini, J. Fluid Mech. 581, 167-197 (2007)

9. Y. Renard, J. Pommier, http://home.gna.org/getfem (2010)

10. P.R. Amestoy, I.S. Duff, J.L' Excellent, J. Koster, Applied Parallel Computing. New Paradigms for HPC in Industry and Academia (Springer, 2001), pp. 121-130

11. R.B. Lehoucq, D.C. Sorensen, C. Yang, SIAM 6 (1998)

12. A.G. Salinger, N.M. Bou-Rabee, R.P. Pawlowski, E.D. Wilkes, E.A. Burroughs, R.B. Lehoucq, L.A. Romero, Sandia National Laboratories, Albuquerque, NM, Technical Report No. SAND2002-0396 (2002)

13. J.O. Pralits, L. Brandt, F. Giannetti, J. Fluid Mech. 650, 513-536 (2010)

14. O. Marquet, D. Sipp, L. Jacquin, J. Fluid Mech. 615, 221-252 (2008) 